\title{
Comparação entre viscossuplementação e plasma rico em plaquetas em lesões condrais de joelhos de pacientes jovens*
}

\section{Platelet-Rich Plasma Versus Hyaluronic Acid for Knee Chondral Injuries In Young Patients}

\author{
Marcus Vinicius Danieli ${ }^{1,2}$ João Paulo Fernandes Guerreiro ${ }^{1,2}$ Telvio Ataide Vimercati ${ }^{10}$ \\ Pedro Henrique Favaro Mendes ${ }^{20}$ Paulo Raphael Tsutomu Katayama Miyazaki2 ${ }^{20}$ \\ Daniele Cristina Cataneo ${ }^{30}$
}

${ }^{1}$ Grupo de Cirurgia do Joelho, Uniorte Hospital de Ortopedia, Londrina, PR, Brasil

2 Hospital Evangélico de Londrina, Londrina, PR, Brasil

${ }^{3}$ Departamento de Cirurgia, Universidade Estadual Paulista (UNESP), Faculdade de Medicina, Botucatu, Botucatu, SP, Brasil

\author{
Endereço para correspondência Marcus Vinicius Danieli, PhD, \\ Uniorte Hospital de Ortopedia, Av. Higienópolis, 2600, Londrina, PR, \\ 86050-000, Brasil (e-mail: mvdanieli@hotmail.com).
}

Rev Bras Ortop 2021;56(5):634-640.

\begin{abstract}
Resumo
Palavras-chave

- ácido hialurônico

- cartilagem articular

- joelho

- plasma rico em plaquetas

- viscossuplementação

Objetivo Comparar o resultado clínico e funcional da aplicação de ácido hialurônico (AH) ou plasma rico em plaquetas (PRP) no tratamento de pacientes jovens portadores de lesões condrais em joelhos, sem artrose.

Métodos Avaliação clínica e funcional prospectiva de 30 pacientes adultos jovens, com lesão condral no joelho, submetidos a tratamento conservador, com aplicação de AH ou PRP, com seguimento mínimo de 12 meses. Para avaliação, foi utilizado o Western Ontário and McMaster Universities Arthritis Index (WOMAC) e a escala visual analógica (EVA) da dor.

Resultados Avaliado pelo questionário de WOMAC, o grupo PRP mostrou melhora em todos os pontos de avaliação com significância estatística, já o grupo AH não mostrou melhora nos escores. Com relação à EVA, o PRP também mostrou melhora em todos os pontos de avaliação, e o grupo AH mostrou melhora com 6 e 12 meses. Quando comparados, o grupo PRP foi melhor que o grupo $\mathrm{AH}$ em todos os pontos de avaliação, de acordo com a escala de WOMAC, e até 6 meses nos resultados da EVA.

Conclusão O PRP obteve melhor resultado clínico e funcional quando aplicado em joelhos com lesões condrais de pacientes jovens, sem artrose, avaliado pelo questionário de WOMAC e pela EVA. Este resultado se manteve até 12 meses.

Nível de evidência Ensaio clínico randomizado (Tipo 2B)
\end{abstract}

\footnotetext{
Trabalho desenvolvido no Grupo de Cirurgia do Joelho, Uniorte Hospital de Ortopedia, Londrina, PR, Brasil.
}

recebido

13 de Abril de 2020

aceito

17 de Setembro de 2020

Publicado on-line

Abril 19, 2021
DOI https://doi.org/ $10.1055 / \mathrm{s}-0041-1724075$. ISSN 0102-3616.
(C) 2021. Sociedade Brasileira de Ortopedia e Traumatologia. All rights reserved.

This is an open access article published by Thieme under the terms of the Creative Commons Attribution-NonDerivative-NonCommercial-License, permitting copying and reproduction so long as the original work is given appropriate credit. Contents may not be used for commercial purposes, or adapted, remixed, transformed or built upon. (https://creativecommons.org/ licenses/by-nc-nd/4.0/)

Thieme Revinter Publicações Ltda., Rua do Matoso 170, Rio de Janeiro, RJ, CEP 20270-135, Brazil 
Abstract

\section{Keywords}

- hyaluronic acid

- cartilage, articular

- knee

- platelet-rich plasma

- viscosupplementation
Objective The present study aimed to compare the clinical and functional outcomes of hyaluronic acid (HA) or platelet-rich plasma (PRP) applications to treat young patients with knee chondral lesions with no arthrosis.

Methods Prospective clinical and functional evaluation of 30 young adult patients with knee chondral lesions submitted to conservative treatment with HA or PRP for a minimum follow-up time of 12 months. The Western Ontario and McMaster Universities Arthritis Index (WOMAC) and visual analog scale (VAS) were used for the evaluation.

Results According to the WOMAC score, the PRP group showed significant improvement in all evaluated points, whereas the HA group presented no score improvement. In the VAS, the PRP group showed improvement in all evaluated points, and the HA group presented improvement at 6 and 12 months. Compared to the HA group, the PRP group presented better WOMAC scores at all evaluated points and better VAS scores up to 6 months after treatment.

Conclusion Platelet-rich plasma application resulted in better clinical and functional outcomes at both the WOMAC and VAS scores when applied to knees from young patients with chondral lesions, but no arthrosis. These outcomes were sustained for up to 12 months.

Level of evidence Randomized clinical trial (Type 2B)

\section{Introdução}

O tratamento de lesões da cartilagem articular tem sido desafiador para a área ortopédica. Com uma estrutura complexa e bem organizada, o tecido condral tem limitado potencial intrínseco de cicatrização. Lesões por trauma ou por patologias degenerativas frequentemente resultam em deterioração tecidual progressiva, levando à dor articular incapacitante e osteoartrose. $^{1}$

As lesões condrais são comuns no joelho e, em sua maioria, apresentam-se assintomáticas. Widuchowski et al. $^{2}$ demonstraram que lesões condrais podem estar presentes em até $63 \%$ das artroscopias de joelho e classificou $67 \%$ destas como lesões focais. Prakash et al. ${ }^{3}$ afirmaram que mesmo essas lesões focais podem progredir para osteoartrose. Quando sintomáticas, apresentam um amplo espectro de sintomas, variando de somente crepitação esporádica até grande limitação funcional, com dor, derrame articular e incapacidade para atividades básicas do cotidiano.

Diante disso, surge interesse em alternativas conservadoras menos complexas e de menor custo que procedimentos cirúrgicos, como infiltrações articulares com ácido hialurônico $(\mathrm{AH})$ e plasma rico em plaquetas (PRP).

O PRP é uma solução resultante da coleta do sangue, processamento e extração do concentrado de plaquetas que tem sido usado em diversas patologias musculoesqueléticas, e sua natureza autóloga apresenta a vantagem de não oferecer reação imunológica, quando usando o sangue do próprio paciente.

O PRP tem mostrado capacidade de estimular a produção de tecido condral, por meio de fatores de crescimento, e fornece suporte para conexões entre os condrócitos. ${ }^{4}$ Além disso, o PRP age reduzindo a ação pró-inflamatória da interleucina-1, que é reconhecida por ser uma das moléculas que induzem os condrócitos e células sinoviais a produzirem enzimas que degradam a matriz extracelular e aceleram a degeneração na osteoartrose., ${ }^{5,6}$ Outro efeito é por meio de fatores de crescimento presentes nas plaquetas, como o transforming growth factor beta (TGF- $\beta$ ), ${ }^{7,8}$ com ação condrogênica sobre os condrócitos, inibindo a apoptose dessas células e induzindo a produção de colágeno. ${ }^{9,10}$

Já o ácido hialurônico $(\mathrm{AH})$ é um componente básico da cartilagem articular e do líquido sinovial. É composto de repetidas sequências de ácido glicurônico e $\mathrm{N}$-acetilglicosamina. Os sinoviócitos tipo B sintetizam e secretam $\mathrm{AH}$, que tem entre suas funções: promover proliferação e diferenciação de condrócitos ${ }^{11}$ e regular, inibindo, citocinas pró-inflamatórias, como a interleucina 8 (IL-8) e a transforming growth factor alpha $(\mathrm{TNF}-\alpha) .{ }^{12}$ Como agente farmacológico, o AH já mostrou ter efeito anabólico, anti-inflamatório e antinociceptivo sobre o tecido condral. ${ }^{13}$ A molécula de $\mathrm{AH}$ também tem alta capacidade viscoelástica, higroscópica e de biocompatibilidade. Diante dessas propriedades, o $\mathrm{AH}$ funciona como um ótimo lubrificante, absorvedor de impacto, e age no balanço hídrico, regulando fluídos. ${ }^{14}$

Portanto, vislumbrando além das tradicionais opções de tratamento conservador e cirúrgico, este estudo busca avaliar a eficácia clínica e funcional das infiltrações de PRP e de AH em lesões condrais, uma vez que há poucas pesquisas relacionadas ao uso destes em pacientes jovens com estas lesões em estágio de pré-artrose.

\section{Materiais e métodos}

O presente estudo foi aprovado pelo Comitê de Ética e Pesquisa (CAAE:58459816.5.0000.0099), e todos os indivíduos selecionados concordaram em participar por meio da assinatura de 
termo de consentimento livre e esclarecido (TCLE). Assim, foi realizado um estudo longitudinal prospectivo, em que foram acompanhados 30 pacientes com lesões de cartilagem diagnosticadas por meio de ressonância nuclear magnética (RNM) e classificadas pela International Cartilage Repair Society (ICRS).

Os critérios de inclusão para o estudo foram pacientes com lesão condral em joelho, até o grau III da classificação da ICRS, entre 18 e 50 anos de idade, com proposta de tratamento conservador que incluía viscossuplementação ou infiltração intra-articular de PRP. A distribuição dos pacientes entre os dois grupos foi feita de forma aleatória, por meio de sorteio. Todos os pacientes tentaram, antes das aplicações, no mínimo 3 meses de tratamento conservador com fisioterapia e medicação analgésica sem sucesso.

Os critérios de exclusão foram pacientes com idade superior a 50 ou abaixo de 18 anos, presença de artrose (classificação de Kellgreen \& Lawrence acima de II no exame radiográfico), índice de massa corpórea (IMC) $\geq 30$ ou pacientes que não aceitaram participar do estudo. ${ }^{14}$ Os pacientes não poderiam ter usado anti-inflamatórios não esteroidais por 3 semanas antes das aplicações.

O grupo PRP foi formado por 15 pacientes, submetidos à aplicação intra-articular de PRP. A coleta do sangue do paciente foi feita sempre no dia de cada infiltração.

O PRP foi colhido e preparado de maneira estéril, seguindo os critérios exigidos para manipulação de derivados do sangue, ambulatorialmente, por uma bioquímica experiente na técnica. Uma veia periférica foi puncionada e foi feita a coleta de $20 \mathrm{ml}$ de sangue em tubos estéreis de coleta a vácuo de $5 \mathrm{ml}$ com $10 \%$ de citrato de sódio. Os tubos foram, então, centrifugados a 1.200 RPM por 10 minutos à temperatura ambiente, em uma centrifuga com $6,5 \mathrm{~cm}$ de raio, da marca Fanem ${ }^{\circledR}$ (Guarulhos, São Paulo, SP, Brasil). O resultado dessa centrifugação é composto por três camadas: células vermelhas (fundo do tubo), células brancas (fina camada sobre as hemácias, chamada de buffy coat) e plasma (camada mais superficial). O plasma era, a seguir, transferido para outro tubo de ensaio estéril de $10 \mathrm{ml}$, sem o buffy coat, sendo submetido a nova centrifugação por 5 minutos na mesma velocidade de rotação da primeira. O resultado desta segunda centrifugação são duas camadas, sendo a metade superior plasma pobre em plaquetas e a metade inferior, o PRP ou também chamado de concentrado de plaquetas. Este PRP (pobre em leucócitos) extraído foi aplicado de maneira intraarticular, uma vez por semana, durante 3 semanas consecutivas. A dose obtida para cada aplicação foi de $2,5 \mathrm{ml}$.

Já o grupo $\mathrm{AH}$ foi composto por 15 pacientes submetidos à viscossuplementação com $\mathrm{AH}$. A injeção era feita uma vez por semana, durante 3 semanas consecutivas, com o produto de nome comercial SupraHyal Duo 2,5 mg/2,5 ml (Tedec-Meiji Farma S.A.; Madri, Espanha - importado por Zodiac Produtos Farmacêuticos S/A).

Os pacientes foram avaliados por meio da aplicação do questionário Western Ontário and McMaster Universities Arthritis Index (WOMAC) e da escala visual analógica (EAV) da dor. Os questionários eram aplicados 5 vezes em cada paciente, considerando os seguintes tempos: antes da $1^{\text {a }}$ infiltração, no dia da $3^{\mathrm{a}}$ infiltração e no $3^{\circ}, 6^{\circ}$ e $12^{\circ}$ mês a partir do início do tratamento.
Para as comparações estatísticas, foi proposto o modelo de regressão linear com efeitos mistos (efeitos aleatórios e fixos). Os modelos lineares de efeitos mistos são utilizados na análise de dados em que as respostas estão agrupadas (mais de uma medida para um mesmo indivíduo) e a suposição de independência entre as observações num mesmo grupo não é adequada. Para as comparações, foi utilizado o pós-teste por contrastes ortogonais. As análises foram realizadas por meio do software SAS 9.2 (SAS Institute, Cary, NC, EUA). Para todas as comparações adotou-se o nível de significância de $5 \%$.

O fluxograma do estudo está representado na -Figura 1.

\section{Resultados}

Entre os 15 pacientes inicialmente submetidos à infiltração de PRP, um paciente optou por descontinuar o tratamento após a primeira dose, devido a dor intensa e derrame articular no joelho. 0 quadro se desenvolveu nas primeiras 24 horas após o procedimento e teve resolução total em 3 dias. Esse paciente foi excluído do trabalho, deixando o grupo PRP com 14 pacientes. Sintomas semelhantes ocorreram em outros 2 pacientes deste grupo, que optaram por continuar no estudo e realizar as duas infiltrações seguintes, após as quais não houve recidiva da dor.

Quanto à constituição, o grupo PRP possuía 11 homens e 3 mulheres e teve média de idade de 34,93 anos (com idade mínima de 18 anos e máxima de 50 anos). Já o grupo $\mathrm{AH}$ foi composto por 11 pacientes do sexo masculino e 4 do sexo feminino, sendo a média de idade 36,47 anos (com idade mínima de 22 anos e máxima de 50 anos). Com relação a lateralidade, idade e sexo, os grupos foram homogêneos estatisticamente ( $\mathbf{- T a b e l a ~} \mathbf{1}$ ).

Com relação aos resultados do questionário WOMAC, os escores médios, desvio padrão, mediana, valores mínimos e máximos dos grupos são apresentados na - Tabela 2. O grupo PRP mostrou melhora dos escores, com significância estatística em todos os pontos de avaliação quando comparados a antes do tratamento. $\mathrm{O}$ grupo $\mathrm{AH}$ não mostrou melhor resultado em nenhum momento neste quesito. Quando comparados os tratamentos, o grupo PRP foi melhor em todos os pontos de avaliação do que o grupo $\mathrm{AH}$ (-Tabelas 2 e $\mathbf{3}$ ).

Quanto aos resultados da EVA, os escores médios, desvio padrão, mediana, valores mínimos e máximos dos grupos são apresentados na - Tabela 4. O grupo PRP, também nesta variável, mostrou melhora em todos os pontos de avaliação. Já o grupo AH mostrou melhora com 6 e 12 meses apenas. Na comparação entre grupos, o PRP teve melhor resultado neste quesito no pós-aplicação, com 3 e 6 meses (-Tabelas 4 e 5 ).

\section{Discussão}

O PRP tem sido usado para tratamento de artrose com bons resultados. ${ }^{15-20}$ Esses efeitos são ainda melhores quanto menor a idade dos pacientes e mais leve o acometimento articular. ${ }^{17,21-23}$ Porém ainda não havia estudo avaliando a aplicação para lesões condrais focais sem artrose no joelho.

Em uma meta-análise recente, Chen et al. ${ }^{24}$ concluíram que o PRP proporciona maior alívio da dor e melhora 


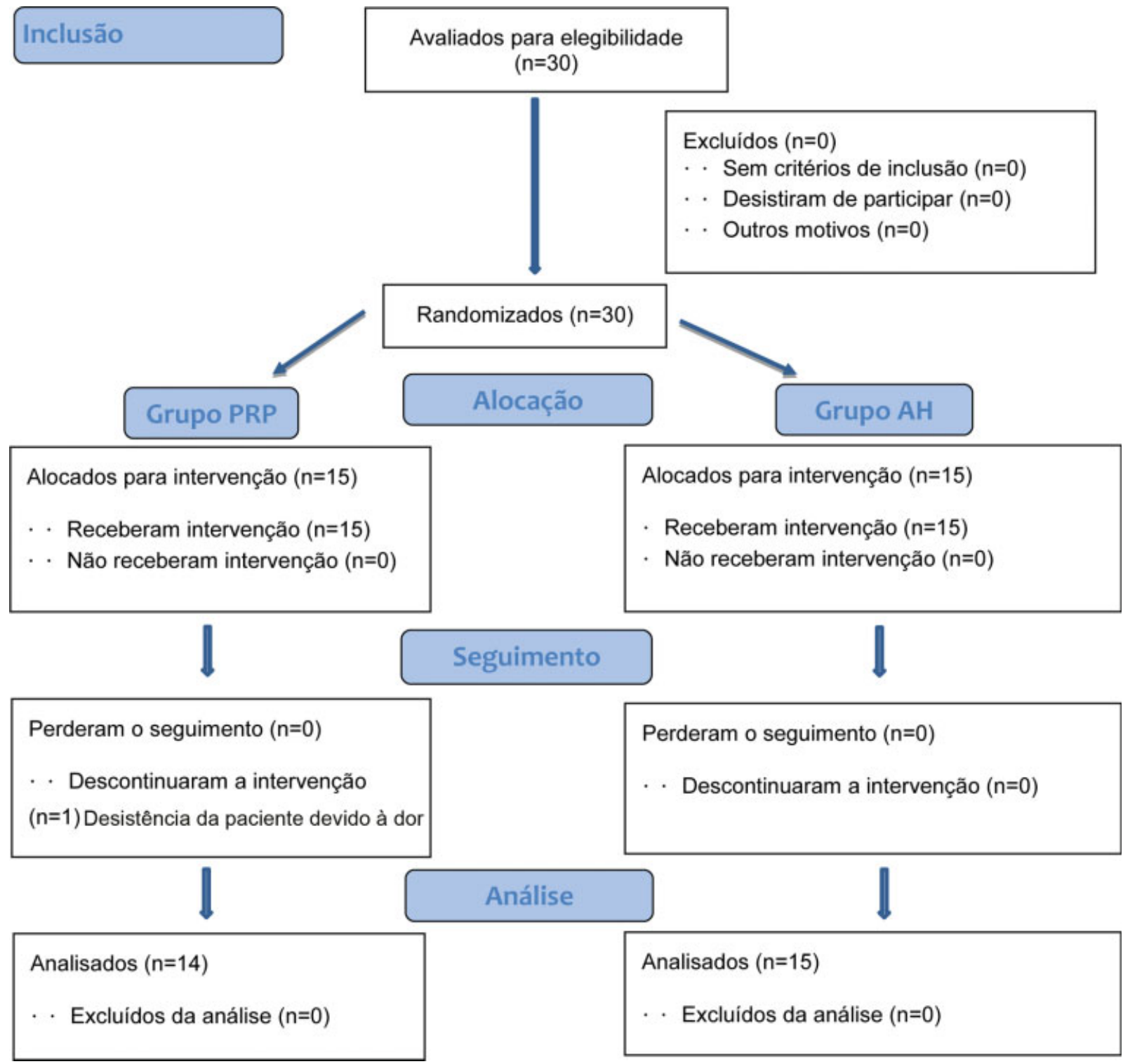

Fig. 1 Fluxograma do estudo.

Tabela 1 Perfil dos pacientes distribuídos entre os grupos

\begin{tabular}{|l|l|l|}
\hline GRUPO & AH & PRP \\
\hline $\mathrm{N}$ & 15 & 14 \\
\hline $\begin{array}{l}\text { Média de idade } \\
\text { (mínimo e máximo) }\end{array}$ & $\begin{array}{l}36,47 \\
(22-50)\end{array}$ & $\begin{array}{l}34,93 \\
(18-50)\end{array}$ \\
\hline Sexo F & 4 & 3 \\
\hline Sexo M & 11 & 11 \\
\hline Lado D & 7 & 3 \\
\hline Lado E & 3 & 8 \\
\hline Bilateral & 5 & 4 \\
\hline
\end{tabular}

Abreviaturas: AH, ácido hialurônico; D: direito; E: esquerdo; F: feminino; M: masculino; PRP, plasma rico em plaquetas.

funcional, no período de 12 meses, quando comparado ao $\mathrm{AH}$ e ao placebo em pacientes com osteoartrose de joelho. Esse estudo também indica que não há diferença quanto ao risco de efeitos adversos entre os métodos. Contudo, o autor destacou a heterogeneidade tanto na preparação do PRP, quanto à gravidade da artrose. Nossos resultados foram semelhantes; no entanto, a novidade foi o uso em pacientes sem artrose e jovens, ampliando as opções de uso desta terapia.

Ainda não se sabe a melhor dose para o PRP, mas estudos sugerem que quanto mais repetições das aplicações, melhor o resultado funcional. ${ }^{15,19,21}$ Em nosso estudo, optamos por 3 aplicações, para ser igual à dose sugerida do produto que usamos na viscossuplementação. Também não se sabe qual concentração de plaquetas seria melhor, discutindo-se até que, na verdade, teríamos que avaliar e quantificar o melhor fator de crescimento ou citocina presente no PRP para cada tecido alvo. ${ }^{18,19,25,26}$

Os leucócitos podem causar efeitos adversos, tais como artrite reacional, além de poder estimular o catabolismo condral. ${ }^{23,27}$ Por isso, optamos, em nosso estudo, pelo uso de PRP pobre em leucócitos, o que pode ter influenciado nos resultados a favor do PRP. 
Tabela 2 Valores do questionário WOMAC para os grupos (média, desvio padrão, mediana, valores mínimos e máximos)

\begin{tabular}{|c|c|c|c|c|c|c|}
\hline \multicolumn{7}{|l|}{ WOMAC } \\
\hline GRUPO & Tempo & Média & Desvio padrão & Mínimo & Mediana & Máximo \\
\hline \multirow[t]{5}{*}{ PRP } & Pré & 34,78 & 16,99 & 1 & 37,5 & 61 \\
\hline & Pós & 20,56 & 13,56 & 1 & 17,0 & 50 \\
\hline & $3 \mathrm{~m}$ & 16,94 & 10,32 & 1 & 13,5 & 45 \\
\hline & $6 \mathrm{~m}$ & 16,39 & 10,81 & 1 & 14,5 & 40 \\
\hline & $12 \mathrm{~m}$ & 19,33 & 13,86 & 1 & 16,0 & 48 \\
\hline \multirow[t]{5}{*}{ AH } & Pré & 23,95 & 17,48 & 2 & 20,5 & 62 \\
\hline & Pós & 21,35 & 21,18 & 1 & 15,0 & 79 \\
\hline & $3 \mathrm{~m}$ & 24,45 & 25,96 & 2 & 14,5 & 85 \\
\hline & $6 \mathrm{~m}$ & 21,80 & 19,69 & 1 & 13,5 & 63 \\
\hline & $12 \mathrm{~m}$ & 25,80 & 19,68 & 2 & 21,5 & 63 \\
\hline
\end{tabular}

Abreviaturas: AH, ácido hialurônico; WOMAC, Western Ontário and McMaster Universities Arthritis Index.

Tabela 3 Comparação entre os grupos e tempos dos resultados do questionário de WOMAC

\begin{tabular}{|l|l|l|}
\hline Comparações & Estimativa & Valor de $P$ \\
\hline PRP (pré e pós) & 14,22 & $<0,01$ \\
\hline PRP (pré e 3 meses) & 17,83 & $<0,01$ \\
\hline PRP (pré e 6 meses) & 18,39 & $<0,01$ \\
\hline PRP (pré e 12 meses) & 15,44 & $<0,01$ \\
\hline AH (pré e pós) & 2,6 & 0,34 \\
\hline AH (pré e 3 meses) & $-0,5$ & 0,85 \\
\hline AH (pré e 6 meses) & 2.15 & 0,43 \\
\hline AH (pré e 12 meses) & $-1,85$ & 0,50 \\
\hline Pós (PRP x AH) & 15,70 & $<0,01$ \\
\hline 3 meses (PRP x AH) & 16,27 & $<0,01$ \\
\hline 6 meses (PRP x AH) & 16,67 & $<0,01$ \\
\hline 12 meses (PRP x AH) & 17,72 & $<0,01$ \\
\hline
\end{tabular}

Abreviaturas: AH, ácido hialurônico; PRP, plasma rico em plaquetas; WOMAC, Western Ontário and McMaster Universities Arthritis Index.
Han et al., ${ }^{28}$ em sua meta-análise, encontraram resultados similares nas avaliações de WOMAC, EAV e IKDC para PRP e $\mathrm{AH}$, no período entre o $1^{\circ}$ e o $3^{\circ}$ mês após as infiltrações. Entretanto, entre o $6^{\circ}$ e $12^{\circ}$ mês de acompanhamento após infiltração, somente o PRP foi capaz de sustentar a melhora funcional e da dor ocorrida nos primeiros meses. Esses autores ainda destacaram não haver diferença para efeitos adversos entre as duas opções terapêuticas e ressaltou que o PRP tem seus melhores resultados em casos de artrose leve ou moderada.

Di et al., ${ }^{29}$ em revisão sistemática, mostraram que o PRP teria maior capacidade de melhorar o WOMAC por um período mínimo de 24 semanas. Contudo, o mesmo estudo analisou outras mensurações de dor, como IKDC e EVA, e não encontrou diferença estatística em comparação aos grupos submetidos à viscossuplementação. Tavassoli et al. ${ }^{30}$ destacam que, quando comparado a 3 infiltrações de AH por 3 semanas seguidas, somente o PRP foi capaz de melhorar em mais de $30 \%$ os valores de WOMAC. Essa melhora foi significativamente maior quando feitas duas doses de PRP com

Tabela 4 Valores da EVA para os grupos (média, desvio padrão, mediana, valores mínimos e máximos)

\begin{tabular}{|c|c|c|c|c|c|c|}
\hline \multicolumn{7}{|c|}{ ESCALA DE DOR } \\
\hline GRUPO & Tempo & Média & Desvio padrão & Mínimo & Mediana & Máximo \\
\hline \multirow[t]{5}{*}{ PRP } & Pré & 6,06 & 2,62 & 0 & 6,0 & 10 \\
\hline & Pós & 4,50 & 2,36 & 0 & 5,0 & 8 \\
\hline & $3 \mathrm{~m}$ & 4,50 & 2,36 & 0 & 5,0 & 8 \\
\hline & $6 \mathrm{~m}$ & 3,67 & 2,11 & 0 & 4,5 & 7 \\
\hline & $12 \mathrm{~m}$ & 4,06 & 1,76 & 1 & 4,5 & 6 \\
\hline \multirow[t]{5}{*}{$\mathrm{AH}$} & Pré & 5,95 & 2,58 & 0 & 6,0 & 10 \\
\hline & Pós & 5,20 & 3,12 & 0 & 5,5 & 10 \\
\hline & $3 \mathrm{~m}$ & 5,20 & 3,40 & 0 & 5,0 & 10 \\
\hline & $6 \mathrm{~m}$ & 4,85 & 2,96 & 0 & 5,0 & 10 \\
\hline & $12 \mathrm{~m}$ & 4,50 & 2,31 & 1 & 5,0 & 8 \\
\hline
\end{tabular}

Abreviaturas: AH, ácido hialurônico; EVA, escala visual analógica; PRP, plasma rico em plaquetas. 
Tabela 5 Comparação entre os grupos e tempos dos resultados da EVA

\begin{tabular}{|l|l|l|}
\hline Comparações & Estimativa & Valor de $P$ \\
\hline PRP (pré e pós) & 1,56 & $<0,01$ \\
\hline PRP (pré e 3 meses) & 1,56 & $<0,01$ \\
\hline PRP (pré e 6 meses) & 2,39 & $<0,01$ \\
\hline PRP (pré e 12 meses) & 2,00 & $<0,01$ \\
\hline AH (pré e pós) & 0,75 & 0,09 \\
\hline AH (pré e 3 meses) & 0,75 & 0,09 \\
\hline AH (pré e 6 meses) & 1,10 & 0,01 \\
\hline AH (pré e 12 meses) & 1,45 & $<0,01$ \\
\hline Pós (PRP x AH) & 2,12 & $<0,01$ \\
\hline 3 meses (PRP x AH) & 2,12 & $<0,01$ \\
\hline 6 meses (PRP x AH) & 1,98 & 0,02 \\
\hline 12 meses (PRP x AH) & 1,24 & 0,14 \\
\hline
\end{tabular}

Abreviaturas: AH, ácido hialurônico; EVA, escala visual analógica; PRP, plasma rico em plaquetas.

intervalo de 3 semanas, em comparação ao grupo em que foi feita somente uma dose.

Apesar de o uso clínico rotineiro do PRP intra-articular ainda não ser uma realidade no Brasil, sendo liberada sua aplicação apenas em pesquisas, no presente estudo esse método se mostrou eficaz em pacientes jovens com lesões condrais de joelho, ao reduzir as limitações de atividades diárias - avaliado por meio do questionário de WOMAC, e também ao diminuir a dor global do paciente - medida pela EVA.

Por outro lado, o AH mostrou melhora apenas após 6 meses da aplicação, durando até 12 meses quando avaliado pela EVA, e menor consistência de resultados quanto ao WOMAC. Há de se destacar ainda que tanto o PRP quanto o $\mathrm{AH}$ possuem variação quanto à sua fórmula, com divergentes formas de preparação e concentrações de plaquetas no primeiro método e, principalmente, os diferentes pesos moleculares entre as soluções comercializadas para viscossuplementação.

Como limitações deste estudo, podemos citar o número limitado de pacientes. Essa limitação se deu pela dificuldade do uso do PRP em nosso meio, devido a restrições legais, e pela limitação do uso da viscossuplementação, devido a custos. A ausência de grupo controle ou sham também pode ser uma fonte de viés. A ausência de cegamento pode ser citada como limitação, tendo sido optada para evitar retirada de sangue do paciente randomizado para o grupo $\mathrm{AH}$.

Portanto, além das medidas conservadoras, como educação do paciente quanto à sua lesão e limitações, orientações de atividade física para fortalecimento muscular, perda ponderal, uso de analgésicos e anti-inflamatórios, deve-se considerar ambos os métodos para o tratamento de lesões condrais de joelho em pacientes jovens. Ressalta-se, contudo, a necessidade de mais estudos em pacientes com esse perfil de lesão para melhor embasamento científico.

\section{Conclusão}

Com base nos resultados obtidos podemos concluir que o PRP obteve melhor resultado clínico e funcional quando aplicado em joelhos de pacientes jovens com lesões condrais, sem artrose, avaliado pelo questionário de WOMAC e EVA. Este resultado se manteve até 12 meses.

\section{Suporte Financeiro}

Não houve suporte financeiro de fontes públicas, comerciais, ou sem fins lucrativos.

\section{Conflito de interesses}

Os autores declaram inexistência de conflito de interesses na realização deste trabalho.

\section{Referências}

1 Lories RJ, Luyten FP. The bone-cartilage unit in osteoarthritis. Nat Rev Rheumatol 2011;7(01):43-49

2 Widuchowski W, Widuchowski J, Trzaska T. Articular cartilage defects: study of 25,124 knee arthroscopies. Knee 2007;14(03): 177-182

3 Prakash D, Learmonth D. Natural progression of osteo-chondral defect in the femoral condyle. Knee 2002;9(01):7-10

4 Sánchez M, Anitua E, Orive G, Mujika I, Andia I. Platelet-rich therapies in the treatment of orthopaedic sport injuries. Sports Med 2009;39(05):345-354

5 van Buul GM, Koevoet WLM, Kops N, et al. Platelet-rich plasma releasate inhibits inflammatory processes in osteoarthritic chondrocytes. Am J Sports Med 2011;39(11):2362-2370

6 Sundman EA, Cole BJ, Karas V, et al. The anti-inflammatory and matrix restorative mechanisms of platelet-rich plasma in osteoarthritis. Am J Sports Med 2014;42(01):35-41

7 Sánchez M, Anitua E, Azofra J, Aguirre JJ, Andia I. Intra-articular injection of an autologous preparation rich in growth factors for the treatment of knee OA: a retrospective cohort study. Clin Exp Rheumatol 2008;26(05):910-913

8 Lubkowska A, Dolegowska B, Banfi G. Growth factor content in PRP and their applicability in medicine. J Biol Regul Homeost Agents 2012;26(02 Suppl 1):3S-22S

9 Pavlovic V, Ciric M, Jovanovic V, Stojanovic P. Platelet Rich Plasma: a short overview of certain bioactive components. Open Med (Wars) 2016;11(01):242-247

10 Parrish WR, Roides B. Platelet rich plasma in osteoarthritis: more than a growth factor therapy. Musculoskelet Regen 2017;3:e1518

11 Kawasaki K, Ochi M, Uchio Y, Adachi N, Matsusaki M. Hyaluronic acid enhances proliferation and chondroitin sulfate synthesis in cultured chondrocytes embedded in collagen gels. J Cell Physiol 1999;179(02):142-148

12 Wang CT, Lin YT, Chiang BL, Lin YH, Hou SM. High molecular weight hyaluronic acid down-regulates the gene expression of osteoarthritis-associated cytokines and enzymes in fibroblastlike synoviocytes from patients with early osteoarthritis. Osteoarthritis Cartilage 2006;14(12):1237-1247

13 Ghosh P. Osteoarthritis and hyaluronan-palliative or disease-modifying treatment? Semin Arthritis Rheum 1993;22(06 Suppl 1):1-3

14 Laurent TC, Laurent UBG, Fraser JRE. The structure and function of hyaluronan: An overview. Immunol Cell Biol 1996;74(02):A1-A7

15 Kavadar G, Demircioglu DT, Celik MY, Emre TY. Effectiveness of platelet-rich plasma in the treatment of moderate knee osteoarthritis: a randomized prospective study. J Phys Ther Sci 2015;27(12):3863-3867 
16 Meheux CJ, McCulloch PC, Lintner DM, Varner KE, Harris JD. Efficacy of Intra-articular Platelet-Rich Plasma Injections in Knee Osteoarthritis: A Systematic Review. Arthroscopy 2016;32 (03):495-505

17 Kanchanatawan W, Arirachakaran A, Chaijenkij K, et al. Shortterm outcomes of platelet-rich plasma injection for treatment of osteoarthritis of the knee. Knee Surg Sports Traumatol Arthrosc 2016;24(05):1665-1677

18 Dai WL, Zhou AG, Zhang H, Zhang J. Efficacy of Platelet-Rich Plasma in the Treatment of Knee Osteoarthritis: A Meta-analysis of Randomized Controlled Trials. Arthroscopy 2017;33(03):659-670.e1

19 Milano G, Sánchez M, Jo CH, Saccomanno MF, Thampatty B, Wang $\mathrm{JH}$. Platelet-rich plasma in orthopaedic sports medicine: state of the art. J ISAKOS 2019;4:188-195

20 Louis ML, Magalon J, Jouve E, et al. Growth Factors Levels Determine Efficacy of Platelets Rich Plasma Injection in Knee Osteoarthritis: A Randomized Double Blind Noninferiority Trial Compared With Viscosupplementation. Arthroscopy 2018;34 (05):1530-1540.e2

21 Görmeli G, Görmeli CA, Ataoglu B, Çolak C, Aslantürk O, Ertem K. Multiple PRP injections are more effective than single injections and hyaluronic acid in knees with early osteoarthritis: a randomized, double-blind, placebo-controlled trial. Knee Surg Sports Traumatol Arthrosc 2017;25(03):958-965

22 Mascarenhas R, Saltzman BM, Fortier LA, Cole BJ. Role of plateletrich plasma in articular cartilage injury and disease. J Knee Surg 2015;28(01):3-10

23 Levy DM, Petersen KA, Scalley Vaught M, Christian DR, Cole BJ. Injections for Knee Osteoarthritis: Corticosteroids, Viscosupple- mentation, Platelet-Rich Plasma, and Autologous Stem Cells. Arthroscopy 2018;34(05):1730-1743

24 Chen P, Huang L, Ma Y, et al. Intra-articular platelet-rich plasma injection for knee osteoarthritis: a summary of meta-analyses. J Orthop Surg Res 2019;14(01):385

25 Oh JH, Kim W, Park KU, Roh YH. Comparison of the Cellular Composition and Cytokine-Release Kinetics of Various PlateletRich Plasma Preparations. Am J Sports Med 2015;43(12): 3062-3070

26 Ha CW, Park YB, Jang JW, Kim M, Kim JA, Park YG. Variability of the Composition of Growth Factors and Cytokines in Platelet-Rich Plasma From the Knee With Osteoarthritis. Arthroscopy 2019;35 (10):2878-2884.e1

27 Riboh JC, Saltzman BM, Yanke AB, Fortier L, Cole BJ. Effect of Leukocyte Concentration on the Efficacy of Platelet-Rich Plasma in the Treatment of Knee Osteoarthritis. Am J Sports Med 2016;44 (03):792-800

28 Han Y, Huang H, Pan J, et al. Meta-analysis Comparing PlateletRich Plasma vs Hyaluronic Acid Injection in Patients with Knee Osteoarthritis. Pain Med 2019;20(07):1418-1429

29 Di Y, Han C, Zhao L, Ren Y. Is local platelet-rich plasma injection clinically superior to hyaluronic acid for treatment of knee osteoarthritis? A systematic review of randomized controlled trials. Arthritis Res Ther 2018;20(01):128

30 Tavassoli M, Janmohammadi N, Hosseini A, Khafri S, Esmaeilnejad-Ganji SM. Single- and double-dose of platelet-rich plasma versus hyaluronic acid for treatment of knee osteoarthritis: A randomized controlled trial. World J Orthop 2019;10(09): $310-326$ 